\title{
Report of the WHO/ISFC task force on the definition and classification of cardiomyopathies ${ }^{\star}$
}

\author{
Definition \\ "Cardiomyopathies are heart muscle diseases of \\ unknown cause".

\section{Classification} \\ Dilated cardiomyopathy \\ Hypertrophic cardiomyopathy \\ Restrictive cardiomyopathy
}

\section{DILATED CARDIOMYOPATHY}

The condition is recognised by dilatation of the left or right ventricle, or both ventricles. Dilatation often becomes severe and is invariably accompanied by hypertrophy. Systolic ventricular function is impaired. Congestive heart failure may or may not supervene. Presentation with disturbances of ventricular or atrial rhythm is common and death may occur at any stage.

\section{HYPERTROPHIC CARDIOMYOPATHY $†$}

This condition is characterised by disproportionate hypertrophy of the left ventricle and occasionally also of the right ventricle which typically involves the septum more than the free wall but occasionally is concentric. Typically the left ventricular volume is normal or reduced. Systolic gradients are common.

Inheritance is usually by an autosomal dominant gene with incomplete penetrance. Characteristic morphological changes, usually most severe in the septum, have been described.

\section{RESTRICTIVE CARDIOMYOPATHY}

This may exist either with or without obliteration. Restrictive cardiomyopathy includes endomyocardial fibrosis and Löffler's cardiomyopathy (endocarditis parietalis fibroplastica). It is proposed

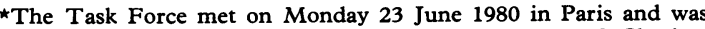
attended by the following: $R$ O Brandenburg, E Chazov, G Cherian, A O Falase, Y Grosgogeat, C Kawai, F Loogen, V Martin Judez, E Orinius, J F Goodwin, E G J Olsen (Chairman), C M Oakley (Rapporteur), Z Pisa (Secretary). Asymmetrical hypertrophy Asymmetric septal hypertrophy, etc. †Pre viously known as:

Idiopathic hypertrophic subaortic stenosis Muscular subaortic stenosis

Obstructive cardiomyopathy

Asymmetrical hypertrophy

Asymmetric septal hypertrophy, etc.
}

that this condition should be referred to as eosinophilic endomyocardial disease.

Endomyocardial scarring usually affects either one or both ventricles, and restricts filling. Involvement of the atrioventricular valves is common but the outflow tracts are spared. Cavity obliteration is characteristic of advanced cases.

"Unclassified cardiomyopathy" covers a few cases which do not fit readily into any group. This includes some with minor abnormalities in which progression to overt cardiomyopathy may or may not occur. This has been referred to as latent cardiomyopathy.

\section{Specific heart muscle disease}

\section{DEFINITION}

Heart muscle disease of known cause or associated with disorders of other systems.

Disorders of the myocardium caused by systemic or pulmonary hypertension, coronary artery disease, valvular heart disease, or congenital cardiac anomalies have been excluded. It was felt that further extension of the classification to include these conditions would have so widened its scope as to render it useless.

\section{Classification}

\section{INFECTIVE}

Viral myocarditis, for example Coxsackie. Rickettsial myocarditis, for example Coxiella. Bacterial, for example staphylococcal. Fungal, for example aspergillus. Protozoal, for example Chagas's. Metazoal, for example filarial.

\section{METABOLIC}

Endocrine

Thyrotoxicosis, hypothyroidism, adrenal cortical insufficience, phaeochromyocytoma, acromegaly.

\section{Familial storage disease and infiltrations}

Haemochromatosis, glycogen storage disease, Hurler's syndrome, Refsum's syndrome, Niemann-Pick disease, Hand-Schüller-Christian disease, FabryAnderson disease, Morquio-Ullrich disease. 


\section{Deficiency}

Disturbances of potassium metabolism, magnesium deficiency, and nutritional disorders such as

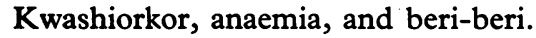

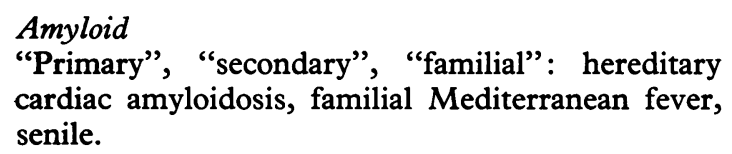
cardiac amyloidosis, familial Mediterranean fever, senile.

GENERAL SYSTEM DISEASES

Connective tissue disorders

Systemic lupus erythematosus, polyarteritis nodosa, rheumatoid arthritis, scleroderma dermatomyositis.

\section{Infiltrations and granulomas}

Sarcoidosis, leukaemia.

HEREDOFAMILIAL

Muscular dystrophies

Duchenne, dystrophia myotonica.

Neuromuscular disorders

Friedreich's ataxia.

\section{SENSITIVITY AND TOXIC REACTIONS}

Sulphonamides, penicillin, antimony, cobalt, emetine, alcohol, isoprenaline, anthracyclines, irradiation, alcohol.

The majority of specific heart muscle diseases are associated with dilatation of the ventricles but there are many exceptions. Localised infiltrations may cause disturbances of rhythm or conduction without generalised myocardial dysfunction. Amyloid infiltration is associated with a unique haemodynamic fault and glycogen storage disease may be associated with a hypertrophic picture.

Alcoholic heart disease. Heart disease may be associated with a heavy alcohol intake. At present we cannot define a causal versus a conditioning role of alcohol nor apply precise diagnostic criteria.

Peripartal heart disease. Heart disease may first manifest in the peripartum period. This is probably a heterogeneous group.

Certain toxins have their main impact on the endomyocardium such as in carcinoid heart disease and methysergide which also affect valves.

Certain heredofamilial disorders such as Noonan's syndrome and lentiginosis may cause a disorder indistinguishable from hypertrophic cardiomyopathy, and Friedreich's ataxia may cause asymmetric hypertrophy with or without dilatation.

Certain disorders do not lend themselves readily to these groups and are listed as "Unclassified". Examples are endocardial fibroelastosis, the infantile form of cardiomyopathy, with histiocytoid change and "Fiedler's myocarditis".

Requests for reprints to Dr C M Oakley, Royal Postgraduate Medical School, Hammersmith Hospital, Du Cane Road, London W12 0HS. 